\title{
L'INFLUENCE DE L'EMPLOI DU LAIT CONCENTRÉ SUR CERTAINES CARACTÉRISTIQUES DES FABRICATIONS FROMAGERES
}

\author{
par Colette TOURNEUR, DO NGOC CU, J. LENOIR \\ et R. VEISSEYRE \\ Laboratoire de technologie, \\ Ecole nationale supérieure agronomique de Grignon.
}

\section{I. - Introduction}

Récemment plusieurs procédés de fabrication de fromages à partir de lait concentré ont été mis au point et étudiés [1, 2]. Leur application présente, entre autres avantages, celui d'accroître le rendement en matière sèche [2]. Les promoteurs de ces procédés ont en outre constaté que les caillés obtenus se comportaient différemment des caillés traditionnels; ainsi le salage en saumure est plus rapide et, au cours de la maturation, le fromage acquiert en un temps plus court l'aspect d'une pâte affinée [3].

Ces différences de rendement et de comportement sont vraisemblablement liées à des différences de composition et de structure des caillés. Il nous a paru utile de réaliser une étude comparative des deux types de fabrications en vue de mettre en évidence certains caractères distinctifs et, si possible, de préciser l'origine de l'augmentation de rendement.

Notre étude a comporté deux grandes étapes. Dans un premier temps nous avons déterminé les caractéristiques analytiques de caillés fabriqués en usine et suivi l'évolution de ces caillés au cours de l'affinage. Dans une seconde série d'essais nous avons réalisé des fabrications de laboratoire et cherché à établir le bilan de ces fabrications.

\section{II. - Méthodes expérimentales}

\section{1) Techniques de fabrication.}

Les caillés étudiés sont du type Carré de l'Est. Chaque série d'essais a comporté une fabrication à partir de lait concentré et une fabrication témoin à partir du lait pasteurisé mis en œuvre pour la concentration.

La première partie du travail a porté sur des fabrications réalisées en usine à partir d'un volume de lait important.

Les fabrications industrielles ont été conduites comme suit :

Le lait, provenant toujours de la même tournée et de la traite du matin, était collecté en citerne. Après pasteurisation à $72^{\circ} \mathrm{C}$ $2^{*}$ 
pendant 60 secondes, une partie du lait (lait-témoin) est refroidie à $8^{\circ} \mathrm{C}$ et stockée à $1^{\circ} \mathrm{C}$ pendant $20-22$ heures. L'autre partie est concentrée dans un appareil à double effet à une température de $40-42^{\circ} \mathrm{C}$ jusqu'à obtention d'un lait à 360 p. 1000 de matière sèche. Ce produit est ensuite refroidi à $13^{\circ} \mathrm{C}$.

Le lait est ensemencé avec un levain, culture mixte de bactéries lactiques sur lait pasteurisé, dont l'acidité est voisine de $80^{\circ}$ Dornic.

Le lait témoin est additionné d'environ 2 p. 100 de levain ( $20 \mathrm{~g}$ pour $120 \mathrm{~g}$ de matière sèche) et sa maturation s'effectue à une température de $34^{\circ} \mathrm{C}$ pendant un temps de $2 \mathrm{~h} 30$ à 3 heures; l'acidité finale est voisine de $21^{\circ}$ Dornic. Le lait concentré reçoit environ $3 \mathrm{p}$. 100 de levain (10 g pour $120 \mathrm{~g}$ de matière sèche), sa maturation est conduite à la température de $13^{\circ} \mathrm{C}$ pendant un temps de 21 à 22 heures, l'acidité atteint environ $60^{\circ}$ Dornic.

Le lait concentré est ensuite additionné d'eau ordinaire en quantité telle que la teneur en matière sèche du lait reconstitué soit de l'ordre de 130 p. 1000 , e'est-à-dire supérieure d'environ 10 p. 100 à celle du lait témoin. Le lait reconstitué est alors additionné d'une nouvelle dose de levain (10 g pour $120 \mathrm{~g}$ de matière sèche) et il subit une maturation complémentaire à $34^{\circ} \mathrm{C}$ pendant le temps nécessaire à l'obtention d'une acidité proche de $24^{\circ}$ Dornie.

Les laits, témoin et reconstitué, sont alors additionnés de chlorure de calcium à la dose de $0,25 \mathrm{~g}$ pour $120 \mathrm{~g}$ de matière sèche, puis emprésurés à la température de $34^{\circ} \mathrm{C}$ par addition de $0,3 \mathrm{ml}$ de présure au 1/10 000 pour $120 \mathrm{~g}$ de matière sèche.

Les opérations de tranchage et de moulage sont réalisées dans les conditions précisées dans le tableau I. Au tranchage le caillé est découpé en morceaux de $15 \times 15 \times 30 \mathrm{~mm}$. Le moulage s'effectue dans les moules classiques des fabrications de Carré de l'Est. $\mathrm{Au}$ cours de l'égouttage dont la durée est de l'ordre de 20 heures on procède à 3 retournements, le premier 20 minutes après moulage, le second après $3 \mathrm{~h} \mathrm{30-4}$ heures, le troisième au bout de 6-7 heures.

Les fabrications de laboratoire, destinées à l'établissement de bilans, ont été conduites selon des modalités identiques, sur des quantités de lait proches de $10 \mathrm{~kg}$, c'est-à-dire des quantités permettant l'obtention de einq caillés de type Carré de l'Est.

\section{2) Méthodes d'analyse.}

a) Analyse des laits et des lactosérums.

Sur les laits mis en œuvre dans les fabrications de laboratoire et sur les lactosérums recueillis les déterminations et dosages suivants ont été réalisés :

- Densité, par aréométrie ;

- Matière sèche, selon la méthode officielle française ; 
- Matière grasse, par la méthode Rose-Gottlieb ;

- Cendres, par incinération du résidu sec à $550^{\circ} \mathrm{C}$;

- Calcium, par précipitation de l'oxalate sur la solution chlorhydrique des cendres et dosage manganimétrique;

- Phosphore inorganique, par réduction du complexe phosphomolybdique sur le filtrat trichloracétique à 12 p. 100 et lecture colorimétrique à $650 \mathrm{~m} \mu$;

- Azote total, par la méthode de Kjeldahl ;

- Azote non protéique, par la méthode de Kjeldahl sur le filtrat trichloracétique à 12 p. 100 ;

- Lactose, par la méthode de Bertrand après défécation au ferrocyanure de zinc ;

- Acide lactique, par mesure de l'acidité titrable.

A partir de certains échantillons de lait des sérums-présure témoins ont été préparés. Après séparation du coagulum par centrifugation, le sérum surnageant est filtré sur papier Whatman $n^{0} 2$ et sa teneur en azote déterminée par la méthode de Kjeldahl.

Enfin, sur certains lactosérums des fabrications de laboratoire, les pertes de caillé ont été estimées. Après 30 à $45 \mathrm{mn}$ de repos le sérum clair superficiel est décanté, la partie inférieure centrifugée (à 5000 tours/mn pendant $10 \mathrm{mn}$ ), le culot est lavé à deux reprises à l'eau distillée puis recueilli et séché à $100^{\circ} \mathrm{C}$ jusqu'à poids constant. La teneur en azote du résidu est déterminée par la méthode de Kjeldahl.

\section{b) Analyse des caillés.}

Sur les caillés, avant salage, les déterminations suivantes ont été effectuées :

- Matière sèche, par la méthode au sable à 102-105० C ;

- Matière grasse, par la méthode Van Gulik pour les caillés de fabrication industrielle et la méthode S.B.R. pour l'établissement des bilans; $105^{\circ} \mathrm{C}$;

- Cendres, par incinération à $550^{\circ} \mathrm{C}$ après dessiccation à 102 -

- Calcium total, selon la même technique que celle utilisée sur le lait ;

- Phosphore inorganique total, par dissociation de la prise d'essai dans l'acide trichloracétique à $12 \mathrm{p}$. 100, filtration et dosage colorimétrique par réduction du complexe phosphomolybdique;

- Calcium et phosphore inorganique solubles, par les méthodes indiquées ci-dessus sur le filtrat d'une suspension aqueuse des caillés ;

- Lactose, par la méthode de Bertrand sur une suspension aqueuse du caillé déféquée par le ferrocyanure de zinc ;

- Acide lactique et lactates, par colorimétrie selon la technique de Steinsholt et Calbert [4] ; 
de $0,5 \mathrm{~g}$;

- Azote total, par la méthode de Kjeldahl sur une prise d'essai

- Azote non protéique, par dissociation du caillé dans l'acide trichloracétique à 12 p. 100, filtration et dosage de l'azote du filtrat selon la méthode de Kjeldahl ;

- $p \mathrm{H}$, par potentiométrie sur une suspension de caillé à 10 p. 100 dans l'eau bidistillée.

En outre, sur les caillés de fabrication industrielle ont été déterminées :

- Les matières azotées solubles selon la technique utilisée pour l'analyse des fromages en cours de maturation ;

- Les matières azotées solubles dénaturables par chauffage $30 \mathrm{mn}$ à $100^{\circ} \mathrm{C}$ du filtrat chlorhydrique à $p \mathrm{H} \mathrm{4,6}$ (matières azotées solubles), filtration et dosage de l'azote du précipité par la méthode de Kjeldahl.

c) Analyse des fromages en cours de maturation.

Sur deux séries d'échantillons fabriqués en usine le même jour, l'une à partir de lait concentré, l'autre à partir du lait témoin, l'évolution des fromages a été suivie au cours de la maturation qui a été conduite dans les mêmes conditions pour chacune des deux séries.

L'analyse a comporté sur chacun des échantillons :

- Une détermination du $p \mathrm{H}$;

- Un dosage des sucres réducteurs et de l'acide lactique ;

- Un fractionnement des matières azotées.

Le fromage est mis en solution dans le citrate de soude [5] et sur cette solution ont été déterminés :

- L'azote total (Kjeldahl);

- L'azote soluble total par précipitation chlorhydrique à $p \mathrm{H}$ 4,6 et dosage de l'azote du filtrat (Kjeldahl).

- L'azote non protéique par précipitation à l'acide trichloracétique à 12 p. 100 et dosage de l'azote du filtrat (Kjeldahl).

\section{III. - Résultats - Discussion}

\section{A) Caractères analytiques des caillés fabriqués industriel- lement.}

Cinq séries de fabrications, comportant chacune une fabrication témoin à partir de lait pasteurisé et une fabrication essai à partir de lait concentré de même origine, ont été réalisées en usine. Les caractéristiques de ces fabrications sont précisées dans le tableau I.

Le lait reconstitué présente une teneur en matière sèche supérieure à celle du lait pasteurisé mais les rapports des teneurs en 
TABLEAU I

PRINCIPALES CARACTÉRISTIQUES DES FABRICATIONS INDUSTRIELLES (1)

\begin{tabular}{|c|c|c|c|c|c|c|c|c|c|c|}
\hline \multirow{2}{*}{ No et type de fabrication } & \multicolumn{2}{|c|}{ No 1} & \multicolumn{2}{|c|}{ No 2} & \multicolumn{2}{|c|}{ No 3} & \multicolumn{2}{|c|}{$\mathrm{N}^{0} 4$} & \multicolumn{2}{|c|}{ No 5} \\
\hline & témoin & essai & témoin & essai & témoin & essai & témoin & essai & témoin & essai \\
\hline \multicolumn{11}{|l|}{ Lait mis en fabrication: } \\
\hline - Matière sèche p. $1000 \ldots \ldots \ldots \ldots$ & 117,7 & 130,1 & 115,2 & 133,0 & 116,0 . & 138,8 & 119,0 & 128,6 & 116,0 & 128,1 \\
\hline - Matière grasse p. $1000 \ldots \ldots \ldots \ldots$ & 32,0 & 34,1 & 30,1 & 37,0 & 31,1 & 41,5 & 33,0 & 36,3 & 31,5 & 34,6 \\
\hline - Matière sèche dégraissée p. $1000 \ldots$ & 85,7 & 96,0 & 85,1 & 96,0 & 84,9 & 97,3 & 86,0 & 92,3 & 84,5 & 93,5 \\
\hline $\begin{array}{l}\text { - Rapport des teneurs en M.S.D. . . } \\
\text { - Acidité titrable (o D) .......... }\end{array}$ & 22,5 & 24,0 & 20,5 & $\begin{array}{l}1,13 \\
24,0\end{array}$ & 20,5 & $\begin{array}{l}1,14 \\
27,0\end{array}$ & 22,5 & $\begin{array}{l}1,07 \\
25,5\end{array}$ & 20,5 & 27 \\
\hline 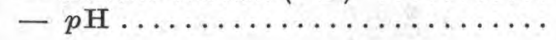 & - & - & 6,38 & 6,30 & 6,20 & 6,10 & 6,28 & 6,20 & 6,39 & 6,19 \\
\hline \multicolumn{11}{|l|}{ Coagulation, égouttage: } \\
\hline - Temps de prise $(\mathrm{mn}) \ldots \ldots \ldots \ldots$ & 5.0 & 4.30 & 5.30 & 4.15 & 6.0 & 3.20 & 4.30 & 3.20 & 6.0 & 4.30 \\
\hline - Temps de tranchage $(\mathrm{mn}) \ldots \ldots \ldots$ & 20. & 20. & 18. & 16. & 20. & 16. & 18. & 15. & 20. & 15. \\
\hline - Temps de moulage (mn) ........ & 75. & 90. & 75. & 80. & 75. & 70. & 75. & 75. & 75. & 75. \\
\hline \multicolumn{11}{|l|}{ Acidification du lactosérum $\left({ }^{\circ} \mathrm{D}\right)$ : } \\
\hline - Acidité au tranchage $\ldots . . \ldots \ldots$ & 16. & 14,5 & 14 & 15 & 14 & 19 & 16 & 16,5 . & 15,5 & 18 \\
\hline - Acidité au moulage ........... & 18 & 16,5 & 17 & 17,5 & 16 & 20,5 & 19,5 & 19,5 & 17 & 20 \\
\hline - Acidité après 4 heures ........ & 47,5 & 46 & 63 & 41 & 70 & 72 & 77 & 77 & 62 & 65 \\
\hline - Acidité après 7 heures ........ & 80 & 81 & 95 & 85 & 95 & 94 & 92 & 97 & 95 & 93 \\
\hline
\end{tabular}

(1) Données établies par les laboratoires des Etablissement Hutin. 
matière sèche des laits reconstitués et des laits témoins n'ont pas une valeur constante; pour les fabrications $1-2-3-4$ et 5 ces rapports sont respectivement de $1,10-1,15-1,19-1,08-$ 1,10. Si l'on envisage les teneurs en matière sèche dégraissée, on observe que leurs rapports présentent des valeurs un peu différentes des précédentes : $1,12-1,13-1,14-1,07-1,11$.

Les acidités du lait témoin et du lait reconstitué sont, compte tenu des rapports de richesse en matière sèche, comparables dans les essais, $n^{0} 1, n^{\circ} 2$, et $n^{0} 4$. Dans les essais $n^{\circ} 3$ et $n^{0} 5$ l'acidité du lait concentré est relativement plus élevée que celle du lait pasteurisé.

Les temps de prise, de tranchage et de moulage mentionnés sur le tableau I sont notés en prenant comme temps zéro le moment de l'emprésurage. L'acidité du lactosérum aux divers stades de l'égouttage permet de constater que l'acidification est comparable dans les fabrications témoins et essais, exception faite des fabrications $n^{\circ} 2$ pour lesquelles on observe un retard notable dans l'acidification du lactosérum provenant de la fabrication du lait concentré.

Pour chaque série de fabrications deux caillés témoins et deux caillés au lait concentré ont été prélevés. Les analyses ont été réalisées en double, sur chacun des caillés en ce qui concerne la matière sèche, la matière grasse, les cendres, le calcium total et soluble, le phosphore inorganique total et soluble, sur le mélange des deux caillés de même origine en ce qui concerne le lactose, l'acide lactique, les matières azotées.

Les caractères analytiques des caillés obtenus sont précisés dans le tableau II. Ces résultats, qui représentent la moyenne des déterminations, permettent de faire les constatations suivantes :

- Les teneurs en matière sèche et en matière sèche non grasse des caillés sont, sinon égales, du moins comparables dans chacune des cinq fabrications. Il apparaît toutefois que les caillés obtenus à partir de lait concentré présentent dans quatre essais des teneurs en matière sèche totale un peu supérieures à celles des cailléstémoins.

- Les taux de cendres sont notablement plus élevés dans les caillés provenant de lait concentré. Les degrés de minéralisation de la matière sèche dégraissée de ces caillés sont augmentés de 15 à 20 p. 100 par rapport aux témoins. Cette augmentation des éléments minéraux dans les fromages de lait concentré apparaît essentiellement due aux sels de calcium et aux phosphates.

Les teneurs en calcium des fabrications-essai sont en effet supérieures d'environ 30 p. 100 à celles des fabrications-témoin dans les essais $1-2-4$ et 5 et de 18 p. 100 dans l'essai no 3 . Rapportées à la matière sèche dégraissée les teneurs en calcium sont augmentées de 25 à 30 p. 100 .

Les mêmes observations peuvent être faites à partir des teneurs 
TABLEAU II

CARACTÈRES ANALYTIQUES DES CAILLÉS FABRIQUÉS INDUSTRIELLEMENT

\begin{tabular}{|c|c|c|c|c|c|c|c|c|c|c|c|}
\hline \multirow{2}{*}{\multicolumn{2}{|c|}{$N^{o}$ et type de fabrication }} & \multicolumn{2}{|c|}{ No 1} & \multicolumn{2}{|c|}{ No 2} & \multicolumn{2}{|c|}{ No 3} & \multicolumn{2}{|c|}{$\mathrm{N}^{\circ} 4$} & \multicolumn{2}{|c|}{ No 5} \\
\hline & & témoin & essai & témoin & essai & témoin & essai & témoin & essai & témoin & essai \\
\hline & $p \mathrm{H}$ & 4,65 & 4,75 & 4,6 & 4,8 & 4,45 & 4,6 & 4,6 & 4,8 & 4,6 & 4,7 \\
\hline \multirow[t]{2}{*}{$\begin{array}{c}\text { p. } 100 \\
\text { du } \\
\text { caillé }\end{array}$} & $\begin{array}{l}\text { Matière sèche } \ldots \ldots \ldots \ldots \\
\text { Matière grasse } \ldots \ldots \ldots \\
\text { Matières minérales } \ldots \ldots \ldots \\
\text { Calcium total. . . . . . . } \\
\text { Calcium soluble } \ldots \ldots \ldots \\
\mathrm{P} \text { inorganique total } \ldots \ldots \\
\text { P inorganique soluble } \ldots \\
\text { Lactose . . . . . . . . . . } \\
\text { Acide lactique et lactates } \\
\text { Matières azotées }(\mathrm{N} \times 6,4)\end{array}$ & $\begin{array}{l}42,3 \\
23,5 \\
1,19 \\
0,29 \\
0,26 \\
0,12 \\
0,10 \\
1,52 \\
0,98 \\
16,8\end{array}$ & $\begin{array}{l}44,2 \\
22 \\
1,44 \\
0,38 \\
0,34 \\
0,155 \\
0,14 \\
1,66 \\
0,97 \\
17,5\end{array}$ & $\begin{array}{l}39,2 \\
20 \\
1,14 \\
0,30 \\
0,28 \\
0,115 \\
0,105 \\
1,46 \\
1,25 \\
14,6\end{array}$ & $\begin{array}{l}40,8 \\
21 \\
1,40 \\
0,39 \\
0,35 \\
0,15 \\
0,14 \\
1,56 \\
1,25 \\
14,4\end{array}$ & $\begin{array}{l}40,7 \\
23 \\
1,16 \\
0,27 \\
0,26 \\
0,105 \\
0,10 \\
1,46 \\
0,99 \\
15,9\end{array}$ & $\begin{array}{l}40,4 \\
22,5 \\
1,31 \\
0,32 \\
0,28 \\
0,125 \\
0,11 \\
1,90 \\
1,00 \\
14,3\end{array}$ & $\begin{array}{l}39,7 \\
23 \\
1,11 \\
0,26 \\
0,25 \\
0,105 \\
0,09 \\
1,51 \\
15,4\end{array}$ & $\begin{array}{l}41,8 \\
23 \\
1,28 \\
0,33 \\
0,28 \\
0,125 \\
0,11 \\
1,70 \\
-\overline{14,5}\end{array}$ & $\begin{array}{l}37,8 \\
20 \\
1,14 \\
0,29 \\
- \\
0,13 \\
- \\
1,66 \\
0,94 \\
14,3\end{array}$ & $\begin{array}{l}39,0 \\
20 \\
1,31 \\
0,37 \\
\overline{0,17} \\
-\overline{01} \\
0,95 \\
13,5\end{array}$ \\
\hline & $\begin{array}{c}\text { Matière sèche dégraissée } \\
\text { (calcul) }(1) \ldots \ldots \ldots \ldots \ldots\end{array}$ & 20,5 & 21,6 & 18,4 & 18,6 & 19,5 & 18,5 & 19,0 & 18,5 & 18,0 & 17,8 \\
\hline $\begin{array}{c}\text { p. } 100 \\
\text { Matière } \\
\text { sèche } \\
\text { dégraissée }\end{array}$ & $\begin{array}{l}\text { Matières minérales } \ldots \ldots \ldots \\
\text { Calcium total } \ldots \ldots \ldots \ldots \\
\text { P inorganique total } \ldots \ldots \\
\text { Lactose. . . . . . . . . } \ldots \ldots \\
\text { Matières azotées }(\mathrm{N} \times 6,4)\end{array}$ & $\begin{array}{l}5,8 \\
1,41 \\
0,58 \\
7,4 \\
82,0\end{array}$ & $\begin{array}{l}6,7 \\
1,76 \\
0,71 \\
7,7 \\
81,0\end{array}$ & $\begin{array}{c}6,2 \\
1,63 \\
0,62 \\
7,9 \\
79,3\end{array}$ & $\begin{array}{l}7,5 \\
2,09 \\
0,80 \\
8,4 \\
77,4\end{array}$ & $\begin{array}{l}5,9 \\
1,38 \\
0,53 \\
7,5 \\
81,5\end{array}$ & $\begin{array}{l}7,1 \\
1,72 \\
0,67 \\
10,3 \\
77,2\end{array}$ & $\begin{array}{l}5,8 \\
1,37 \\
0,55 \\
7,9 \\
81,0\end{array}$ & $\begin{array}{c}6,9 \\
1,78 \\
0,67 \\
9,2 \\
78,3\end{array}$ & $\begin{array}{l}6,3 \\
1,61 \\
0,72 \\
9,2 \\
79,4\end{array}$ & $\begin{array}{l}7,35 \\
2,07 \\
0,95 \\
11,3 \\
75,8\end{array}$ \\
\hline $\begin{array}{l}\text { p. } 100 \\
\mathrm{~N} \text { total }\end{array}$ & Matières azotées solubles. & 12,9 & 10,6 & 9,6 & 10,2 & 11,2 & 12,1 & 10,0 & 11,4 & 9,0 & 11,8 \\
\hline $\begin{array}{c}\text { p. } 100 \\
\mathrm{~N} \text { soluble }\end{array}$ & $\begin{array}{l}\text { Matières azotées dénatura- } \\
\text { bles } \ldots \ldots \ldots \ldots \ldots \ldots \ldots\end{array}$ & 10,1 & 14,5 & 14,3 & 15,0 & 15,2 & 14,5 & 20,1 & 16,3 & 11,7 & 15,1 \\
\hline
\end{tabular}

(1) Matière sèche dégraissée = Matières azotées + Matières minérales + Lactose et acide lactique, 
en phosphore inorganique. Celles des pâtes de lait concentré sont supérieures de 30 p. 100 dans les essais $1-2$ et 5 , de 19 p. 100 dans les essais 3 et 4 . Calculés sur la matière sèche dégraissée les taux de phosphore inorganique sont augmentés de 22 à 32 p. 100. Les augmentations $d u$ phosphore inorganique et du calcium sont donc assez étroitement concordantes.

Sur la base des teneurs en calcium, exprimées en phosphate tricalcique p. 100 de la matière sèche dégraissée, les augmentations de "l'indice de minéralisation" dans les fabrications provenant de lait concentré oscillent entre 0,9 et 1,2 p. 100.

Les rapports des teneurs en calcium et phosphore inorganique solubles des deux types de caillés ont des valeurs voisines de celles des rapports des teneurs en éléments totaux et il ne semble pas y avoir de différences importantes entre les deux fabrications dans les proportions relatives des fractions solubles du calcium et du phosphore inorganique. On constate cependant que les caillés provenant de lait concentré présentent un rapport Ca soluble/Ca total un peu plus faible que les caillés témoin : les rapports moyens et extrêmes sont respectivement 0,87 et $0,84-0,89$ pour les caillés des fabrications au lait concentré, 0,94 et $0,90-0,96$ pour les caillés de fabrications témoin. Cette observation n'est cependant pas confirmée par les rapports phosphore inorganique soluble/phosphore inorganique total.

Les teneurs en lactose des pâtes sont sensiblement plus élevées dans les fabrications de lait concentré. On constate toutefois que les résultats des différents essais ne sont pas tous concordants. Une augmentation appréciable de la proportion de lactose est observée dans les essais 3,4 et 5, mais l'augmentation est peu marquée dans les essais 1 et 2 .

Les teneurs en acide lactique et lactates indiquent que l'acidification des caillés est comparable dans les deux fabrications. Toutefois, le $p \mathrm{H}$ des pâtes obtenues à partir du lait concentré est toujours supérieur à celui des fabrications témoin. Cette différence est sans doute imputable au plus fort degré de minéralisation, et par suite au plus fort pouvoir tampon, des caillés provenant de lait concentré.

La part des matières minérales et du lactose dans la matière sèche dégraissée des caillés provenant de lait concentré étant accrue, la proportion de matières azotées se trouve parallèlement réduite. Il nous a paru intéressant de rechercher si les caillés provenant de lait concentré contiennent une proportion plus grande de protéines du sérum par suite d'une dénaturation au cours du traitement. S'il en était ainsi le pourcentage d'azote soluble par rapport à l'azote total serait diminué. Or, on constate que dans ce type de caillé les proportions d'azote soluble sont plus élevées dans 4 essais sur 5 . En outre, au sein de l'azote soluble les proportions d'azote dénaturable appartenant aux fractions albumine et globuline sont comparables dans les deux types de pâtes. Il ne semble donc pas y avoir 
TABLEAU III

PRINCIPAUX CARACT亡̀RES DISTINCTIFS DES CAILLÉS INDUSTRIELS OBTENUS A PARTIR DE LAIT CONCENTRÉ

\begin{tabular}{|c|c|c|c|c|c|c|}
\hline & & Essai 1 & Essai 2 & Essai 3 & Essai 4 & Essai 5 \\
\hline $\begin{array}{l}\text { Rapports des richesses du caillé } L \text {. } \\
\text { témoin (p. } 100 \text { de la matière sèch }\end{array}$ & $\begin{array}{l}\text { C. et du caillé } \\
\text { dégraissée) }\end{array}$ & & , & & & \\
\hline 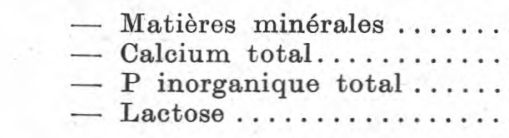 & $\begin{array}{l}\ldots \ldots \ldots \\
\ldots \ldots \ldots \ldots \\
\ldots \ldots \ldots \ldots\end{array}$ & $\begin{array}{l}1,15 \\
1,24 \\
1,22 \\
1,04\end{array}$ & $\begin{array}{l}1,21 \\
1,28 \\
1,29 \\
1,06\end{array}$ & $\begin{array}{l}1,20 \\
1,25 \\
1,26 \\
1,37\end{array}$ & $\begin{array}{l}1,19 \\
1,30 \\
1,22 \\
1,15\end{array}$ & $\begin{array}{l}1,17 \\
1,28 \\
1,32 \\
1,22\end{array}$ \\
\hline Ca soluble/Ca total $\ldots \ldots \ldots \ldots \ldots$ & $\begin{array}{l}\text { Témoin } \ldots \\
\text { L.C. . . . . }\end{array}$ & $\begin{array}{l}0,90 \\
0,89\end{array}$ & $\begin{array}{l}0,93 \\
0,89\end{array}$ & $\begin{array}{l}0,96 \\
0,87\end{array}$ & $\begin{array}{l}0,96 \\
0,84\end{array}$ & - \\
\hline $\mathrm{P}$ inorg. sol./P inorg. total . . . . & $\begin{array}{l}\text { Témoin ... } \\
\text { L.C. . . . . }\end{array}$ & $\begin{array}{l}0,83 \\
0,90\end{array}$ & $\begin{array}{l}0,91 \\
0,93\end{array}$ & $\begin{array}{l}0,95 \\
0,88\end{array}$ & $\begin{array}{l}0,86 \\
0,88\end{array}$ & - \\
\hline $\begin{array}{l}\text { Indice de minéralisation exprimé } \\
\text { en }\left(\mathrm{PO}_{4}\right)_{2} \mathrm{Ca}_{3}-\mathrm{p} .100 \text { de l'extrait } \\
\text { sec dégraissé sur la base des te- } \\
\text { neurs en Ca } \ldots \ldots \ldots \ldots \ldots \ldots \ldots\end{array}$ & $\begin{array}{l}\text { Témoin } \ldots \\
\text { L.C. . . . . . } \\
\text { augmentation }\end{array}$ & $\begin{array}{l}3,64 \\
4,54 \\
0,90\end{array}$ & $\begin{array}{l}4,21 \\
5,40 \\
1,19\end{array}$ & $\begin{array}{l}3,56 \\
4,44 \\
0,88\end{array}$ & $\begin{array}{l}3,54 \\
4,60 \\
1,06\end{array}$ & $\begin{array}{l}4,15 \\
5,34 \\
1,19\end{array}$ \\
\hline
\end{tabular}


de rétention particulière des protéines du sérum dénaturées dans les fromages obtenus à partir de lait concentré.

(Les résultats des analyses de sérum effectuées lors des essais de laboratoire confirment d'ailleurs ce point.)

Les principaux caractères distinctifs des caillés obtenus à partir de lait concentré sont groupés dans le tableau III.

\section{B) Evolution des fromages en cours de maturation.}

Les résultats des déterminations effectuées sur les fabrications de l'essai no 1 , qui ont subi un affinage dans les conditions de la pratique industrielle courante, sont mentionnés dans le tableau IV.

Une constatation s'impose. On ne relève aucune différence appréciable entre les échantillons des fabrications témoin et essai ayant atteint le même stade de maturation. L'évolution du $p H$ et la cinétique de la protéolyse sont pratiquement identiques pour les deux types de fabrications. En ce qui concerne les vitesses de fermentation des sucres et de consommation de l'acide lactique les écarts observés sont faibles et le nombre des données est insuffisant pour attribuer à ces écarts un caractère significatif.

Il ne semble donc pas que les différences observées dans la composition ou dans la structure du caillé se traduisent par un retard ou une accélération notables des phénomènes biochimiques qui président à la maturation de la pâte.

(à suivre)

TABLEAU IV

EVOLUTION DES FROMAGES AU COURS DE LA MATURATION

\begin{tabular}{|c|c|c|c|c|c|c|c|c|c|c|}
\hline & \multicolumn{2}{|c|}{ J. 2} & \multicolumn{2}{|c|}{ J. 8} & \multicolumn{2}{|c|}{ J. 16} & \multicolumn{2}{|c|}{ J. 22} & \multicolumn{2}{|c|}{ J. 29} \\
\hline & témoin & essai & témoin & essai & témoin & essai & témoin & essai & témoin & essai \\
\hline$p H \ldots \ldots \ldots \ldots$ & 4,8 & 5,0 & 5,1 & 5,15 & 5,95 & 5,9 & 6,5 & 6,55 & 6,85 & 6,85 \\
\hline Lactose ....... & 1,60 & 1,66 & 0,7 & 1,2 & 0,15 & 0,25 & traces & tra- & - & - \\
\hline $\begin{array}{l}\text { Acide lactique et } \\
\text { lactates ........ }\end{array}$ & 0,98 & 0,97 & 0,83 & 1,06 & 0,35 & 0,53 & 0,20 & 0,22 & 0,11 & 0,20 \\
\hline 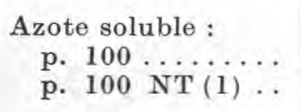 & $\begin{array}{l}0,34 \\
12,9\end{array}$ & $\begin{array}{l}0,29 \\
10,6\end{array}$ & $\begin{array}{l}0,48 \\
17,4\end{array}$ & $\begin{array}{c}0,42 \\
17,6\end{array}$ & $\begin{array}{l}0,60 \\
19,6\end{array}$ & $\begin{array}{c}0,56 \\
19,8\end{array}$ & $\begin{array}{r}0,78 \\
26,4\end{array}$ & $\begin{array}{l}0,70 \\
25,6\end{array}$ & $\begin{array}{l}0,86 \\
27,9\end{array}$ & $\begin{array}{l}0,84 \\
27,5\end{array}$ \\
\hline $\begin{array}{l}\text { Azote non protéique: } \\
\text { p. } 100 \ldots \ldots \ldots \\
\text { p. } 100 \mathrm{NT} \ldots \ldots\end{array}$ & $\begin{array}{l}0,10 \\
4,0\end{array}$ & $\begin{array}{l}0,10 \\
3,8\end{array}$ & $\begin{array}{l}0,19 \\
6,8\end{array}$ & $\begin{array}{l}0,18 \\
7,5\end{array}$ & $\begin{array}{l}0,32 \\
10,4\end{array}$ & $\begin{array}{l}0,29 \\
10,2\end{array}$ & $\begin{array}{l}0,40 \\
13,5\end{array}$ & $\begin{array}{r}0,37 \\
13,7\end{array}$ & $\begin{array}{l}0,48 \\
15,5\end{array}$ & $\begin{array}{l}0,49 \\
16,0\end{array}$ \\
\hline
\end{tabular}

(1) NT : Azote total. 